\title{
Asynchronous MAC Protocol for Spectrum Agility in Wireless Body Area Sensor Networks
}

\author{
Nhat-Quang NHAN, Matthieu GAUTIER and Olivier BERDER \\ IRISA, INRIA, University of Rennes 1, France \\ Email: \{nhat-quang.nhan, matthieu.gautier, olivier.berder\} @ irisa.fr
}

\begin{abstract}
A Wireless Body Area Sensor Network (WBASN) is a special-purpose Wireless Sensor Network (WSN) that supports remote monitoring and entertainment applications. The energy consumption plays an important role in the design of this specific sensor network. Unfortunately, the performance of WBASNs decreases in high interference environments such as the Industrial, Scientific and Medical (ISM) band where wireless spectrums are getting crowded. In this paper, an energy-efficient Medium Access Control (MAC) protocol named C-RICER (Cognitive-Receiver Initiated CyclEd Receiver) is specifically designed for WBASN to cognitively work in high interference environment. C-RICER protocol adapts both transmission power and channel frequency to reduce the interferences and thus, the energy consumption. The protocol is simulated thanks to OMNET++ simulator. Simulation results show that, depending on the interference level, C-RICER is able to outperform the traditional RICER protocol in terms of energy consumption, packet delay, and network throughput.
\end{abstract}

\section{INTRODUCTION}

WBASNs promise many applications in health care, fitness and entertainments. Unlike WSN where sensor nodes are distributed in a wide area, those in WBASNs are interconnected within a human body and interact with different networks for remote monitoring. The amount of sensor nodes in a WBASN is limited and each sensor node has different sensing function. Hence, the network cannot execute data aggregation. Additionally, the typical network topology of a WBASN is centralized since only the center node acts as a data receiver to collect the sensing data from the other nodes. Therefore, it expects high reliability during data sensing and transmission. Moreover, as sensor nodes in WBASNs are distributed among a human body, they require small-size devices, fast data gathering and processing. However, smaller nodes also imply smaller batteries, which create a trade-off between the energy consumption and network performance of WBASNs [1].

Recently, the wireless spectrum in ISM bands is getting crowded. This causes the coexisting interference between the radio users in those open frequency bands including WBASNs. A network operating in high interference environment costs much of energy for the retransmission of data packets. Consequently, the disadvantages of WBASNs in high coexisting interference are high energy consumption, high packet delay, and low network throughput [2].

This paper proposes applying the Cognitive Radio (CR) technique [3][4] to maintain the signal to noise ratio for WBASNs data exchange and therefore overcome the disadvantages of WBASNs in high coexisting interference environment. In this proposition, the WBASN considers to operate in the channel that has less interference among the frequency band when the interference of its current channel reaches a threshold that can cause harmful to the data transmission of the network. To this aim, the WBASN frequently scans for interference level of its current channel to early detect the interference, and only scans the whole frequency band when the switching decision is made. By dynamically scanning and switching among the channels, the WBASN can avoid the coexisting interference. Therefore, it can save energy, reduce packet drop and packet delay. The WSBAN will also include a mechanism that adapts its transmission power when short duration interferences occur.

Since these cognitive tasks are performed by a radio module which is directly controlled by the MAC layer, the design of a cognitive MAC protocol for WBASNs is considered in this paper. Recently, many MAC protocols have been proposed for WBASNs themselves [5]. However, the number of protocol that considers CR into this branch of WSNs is very limited [6]. In contrast, MAC protocols in CR networks have been designed for several applications [7], but no protocol was specifically designed for WBASNs. The contribution of this paper is a cognitive MAC protocol named C-RICER which takes into account WBASN specifications and includes both transmission power and channel frequency adaptation. The performance of C-RICER is compared to the traditional energy-efficient RICER3b protocol thanks to OMNET++ simulator.

This paper is organized as follows. In Section II, the main characteristics of WBASNs that affect the design of C-RICER are pointed out. Section III introduces the related MAC protocols. The algorithm and protocol of C-RICER are presented in Section IV. Simulation results are showed and analyzed in Section V. Finally, a conclusion is drawn in Section VI.

\section{CONTEXT OF WBASN}

The main characteristics of WBASNs could be listed in three folds: network topology, energy distribution and hardware support [8].

First, the typical network topology in WBASNs is the centralized topology. The center node is called coordinator and the other nodes are slaves. Most of times, the coordinator is the only receiver to collect data in a WBASN.

Second, the coordinator in a WBASN normally is cell phone or portable device which is easy to recharge or replace the 


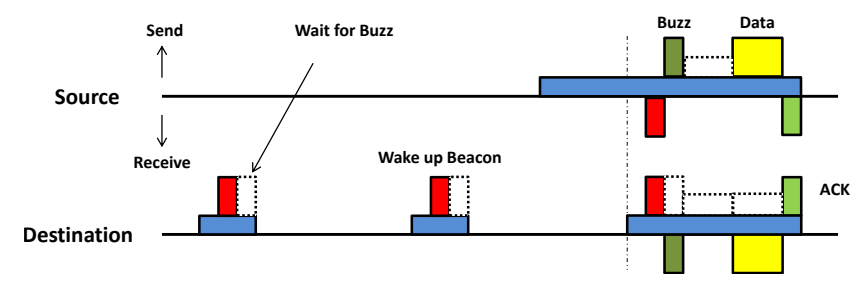

Fig. 1. RICER3b scheme.

battery. Therefore, the coordinator is the only node which is supposed to have less energy constraint compared with (the priority of energy saving is less than) the slaves.

Last, sensor nodes in WBASNs are small-size to ensure the convenience for the patient. Hence, sensor nodes in WBASNs must follow the hardware limitation condition such that only one transceiver is equipped at each sensor (including the coordinator).

The study of C-RICER exploits the centralized network topology for the concept of interference detection. The energy for the cognitive task of C-RICER is mostly consumed at the coordinator. Last but not least, the spectrum agility function of C-RICER considers a very important characteristic of WBASN, where a single transceiver per sensor nodes is used. This single transceiver condition along with energy constraint makes the design of C-RICER in WBASN become more challenging and different from the traditional designs of cognitive MAC protocol [7].

\section{RELATED WORKS}

The main idea of C-RICER design is to include the $\operatorname{cog}$ nitive task in an available energy-efficient MAC protocol in WSN. This section presents the importance of the MAC layer and points out the energy-efficient protocol that is suitable with the context of WBASNs.

In WSN, a great amount of energy is spent in the radio module which is controlled by the MAC layer. Therefore, a good MAC protocol plays a main role in decreasing the energy consumption of the whole network. Traditional MAC protocols can be divided into 2 families depending on how a node gains the medium access: contention-based and reservationbased. Due to the limit of energy and traffic, contention-based is more suitable for WSNs [9]. In contention-based family, receiver-initiated protocols [10] seem more suitable for the context of WBASNs. Using a receiver-initiated protocol, the energy is spent at the receiver more than at the transmitter. As mentioned in Section II, with the centralized network topology, the coordinator is the only receiver in a WBASN and has less energy constraint compared with the slaves. Therefore, using receiver-initiated protocols makes energy for data exchange of WBASNs mostly consumed at the coordinator that matches perfectly with the WBASNs context.

A well-known protocol in this family is Receiver Initiated CyclEd Receiver (RICER) protocol. Fig. 1 illustrates RICER3b protocol. In RICER3b, the Destination node with no data packet to transmit wakes up periodically and transmits a short wake up beacon indicating that it is awake. Then it monitors the channel for a response. If there is no response, the node goes back to sleep. On the other hand, a Source node with data to transmit stays awake, monitors the channel, and waits for a wake up beacon from the Destination.

The use of beacons in RICER3b is different from Zigbee. In Zigbee, the beacons are used to create the synchronization between the super-frames of the nodes. Then, the data is later transmitted using CSMA-CA algorithm based on an appropriate back-off timer. In RICER3b, after receiving wake up beacon, the Source node immediately sends back a buzz signal and starts transmitting data. By receiving this buzz signal, the Destination will stay awake to wait for data from the Source node.

RICER3b shows a great energy and delay efficiency among RICER family [11]. Therefore, RICER3b is utilized as the working algorithm of C-RICER in the low interference channel. Next section will present the algorithm of C-RICER in detail.

\section{C-RICER: A COGNITIVE MAC PROTOCOL}

\section{A. Protocol description}

The general objective of C-RICER is to early detect the interference in its working channel. In C-RICER, channel sensing is periodically performed at the coordinator, the only node that has less energy constraint in the network. According to the detected level of interference, the WBASN can adaptively switch to the lowest interference channel to prevent the coexisting interference as shown in Fig. 2. The sensing and switching is continuously performed during the operation of C-RICER, not only at the initialization as for Zigbee.

In practice, the evolution of wireless channel interferences could be unpredictable. In some case, the interference appears and disappears for a short period. This phenomenon would make the whole network change its working channel frequently and cost much of energy for channel switching [12]. To overcome this, C-RICER includes also the use of power adaptation before channel adaptation is considered.

By using this twofold cognitive functions, C-RICER can maintain the performance of the WBASNs in high interference condition, especially in terms of energy consumption. The working mechanism of C-RICER contains two main tasks which are the data exchange task and the cognitive task. The data exchange task is used for data collection while the cognitive task is used to dynamically adapt both transmission power and channel according to the interference level. The mechanisms of those two tasks are presented in the next two subsections.

\section{B. Network data exchange task}

The data communication of C-RICER is based on RICER3b protocol with the Destination being the coordinator and the Source being the slave nodes. As shown in Fig. 1, its principle consists of five phases:

1) The coordinator wakes up and sends a wake up beacon.

2) The slave receives the wake up beacon and transmits a buzz signal to indicate the coordinator to wait for data. 


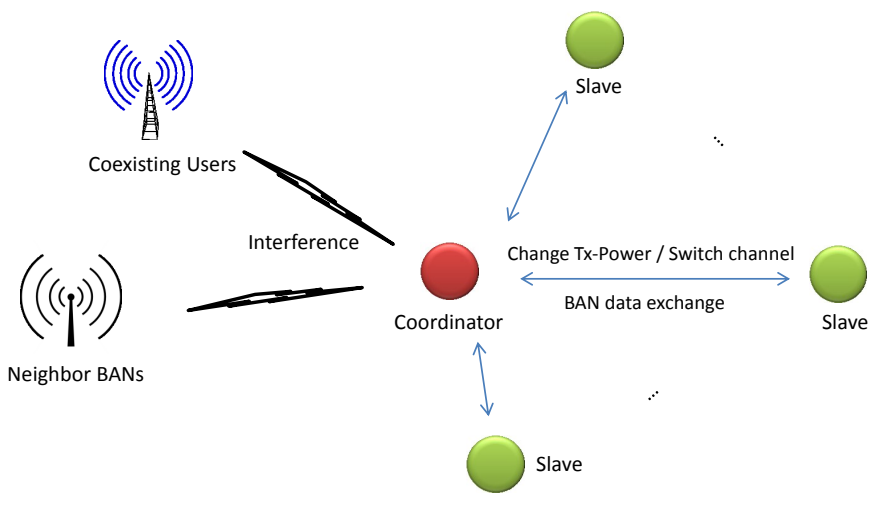

Fig. 2. Overview of C-RICER in WBASN.

3) The coordinator receives buzz and waits data from the slave.

4) The slave sends data and waits ACK from the coordinator.

5) The coordinator receives data and sends ACK to the slave.

\section{Cognitive task}

The cognitive task of C-RICER contains three main features which are: channel sensing, power adaptation and channel adaptation.

1) Channel sensing: Initially, the very important step for the cognitive task is the detection of interference. In this work, the Received Signal Strength Indicator (RSSI) is used to measure the interference level of a wireless channel. In the context of WBASNs, with the purpose of energy saving, the coordinator is the most suitable node in the network to perform the channel sensing because of several reasons.

First, the coordinator is the only node that has less energy constraint in the network. Focusing to exploit the energy at the coordinator instead of slaves can prolong the network lifetime.

Second, when applying the RICER3b scheme to the centralized topology of WBASNs, a communication is only established after the transmitter has successfully received a wake up beacon message from the receiver. The coordinator is considered as the only receiver in a WBASN. Therefore, when the coordinator does channel sensing, there is no com-

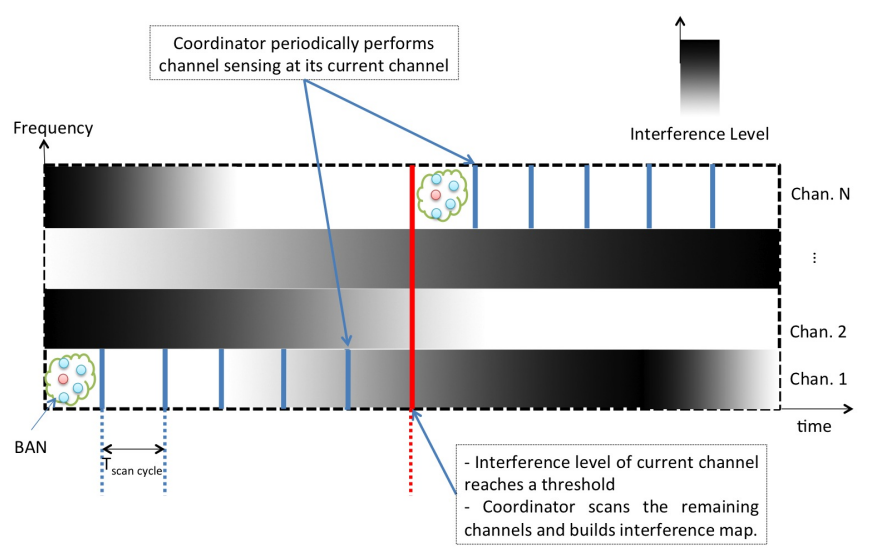

Fig. 3. Channel sensing strategy. munication in that WBASN. The received signal at that time comes only from the interference sources (if existing).

As shown in Fig. 3, because channel sensing and switching cost energy [12], the coordinator of C-RICER periodically senses for interference of only its current working channel instead of the whole frequency band to reduce the sensing energy. Only when the interference level of its current channel is greater than a threshold value, the coordinator will scan for the interference level of the remaining channels to build a full interference map of the frequency band. The period between two consecutive channel sensing activities is $T_{\text {scan cycle }}$.

2) Power adaptation: As stated in the description of CRICER, if only the channel adaptation feature is used, the phenomenon such that the interference only appears for a short duration can make the WBASN switches its channel frequently and cost much of energy for channel switching. For that reason, increasing the transmission power following an appropriate algorithm can not only enhance the signal to noise ratio but also reduce the retransmission in the network. Then, even if the transmitter energy consumption increases with the transmission power, the global energy consumption of WBASN can be reduced due to the reduction of the retransmission.

As shown in Fig. 4, the transceiver at each node is proposed to have two specific transmission powers (TxPowers) which are $P_{1}$ and $P_{2}\left(P_{1}<P_{2}\right)$. Two interference threshold values Thres $_{1}$ and Thres 2 are introduced such that Thres ${ }_{1}<$ Thres $_{2}$. Where packet error rate will increase in proportional to the interference levels of Thres $s_{1}$ and Thres 2 . The value of Thres $_{2}$ will be selected at a point such that the interference starting to effect the packet error rate (when $P_{2}$ is used), but still acceptable for the transmission of control packets.

After each channel sensing, if the interference level (RSSI) of current working channel is less than Thres 1 , the transceiver of each sensor node will work at its default transmission power $P_{1}$. If the coordinator recognizes that the interference level of current channel is greater than Thres $_{1}$ but less than Thres $_{2}$, it will increase TxPower to $P_{2}$. After $P_{2}$ is used, the coordinator will adaptively change its scanning cycle by quickly coming back in $T_{\text {rescan cycle seconds to rescan the channel. After }}$ scanning, if the RSSI of current channel is reduced and less than Thres ${ }_{1}$, the network will go back to use $P_{1}$. Conversely, if the RSSI level is still higher than Thres ${ }_{1}$, the coordinator scans the remaining channels to build the interference map, the whole network will switch to a new channel that has the lowest interference level among the map. The transmission power is also changed to the default value $P_{1}$. This reduces the use of a high TxPower $\left(P_{2}\right)$ for long time which costs energy.

Otherwise, if the RSSI of current working channel is directly greater than Thres $_{2}$, the coordinator also scans the remaining channels to build the interference map, the whole WBASN will switch to the channel that has the lowest interference level in the map immediately without changing transmission power.

The coordinator utilizes the wake up beacon message to broadcast its TxPower information to the slaves. The wake up 


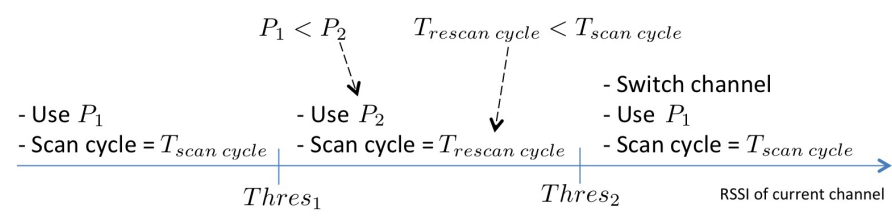

Fig. 4. Power adaptation strategy.

beacon message is added one dedicated bit which contains the information of TxPower level. The slaves will extract this bit from the wake up beacon message. If this bit is zero, $P_{1}$ is used. If not, $P_{2}$ is used.

3) Channel adaptation: Based on the interference map, the WBASN will decide to switch to a new channel when interference level of its current working channel is greater than Thres $_{2}$ or when the interference level of current channel is still high after rescanning. This subsection presents the algorithm used by the C-RICER coordinator to inform the channel switching information to the slaves.

As mentioned, working with a single transceiver makes the channel switching process of the WBASNs become more complicated compared with the traditional cognitive radio networks, where two transceivers are used at each node, one for data exchange and one for sharing the channel information among the sensors [7]. Moreover, the wake up mechanism of the slaves depends on their demand to send the data. Slaves with different sensing functions will wake up randomly and will not receive the switching information from the coordinator at the same time. Therefore, a checklist table is used at the coordinator to count for the slaves that successfully received the switching information. The channel switching algorithm is shown in Fig. 5.

At the coordinator, after the switching decision is made, the index of the channel that has the lowest RSSI among the interference map is attached to a message named Channel Switching Message. Right after that, the coordinator periodically broadcasts the Channel Switching Message to all slaves. After sending the Channel Switching Message, the coordinator

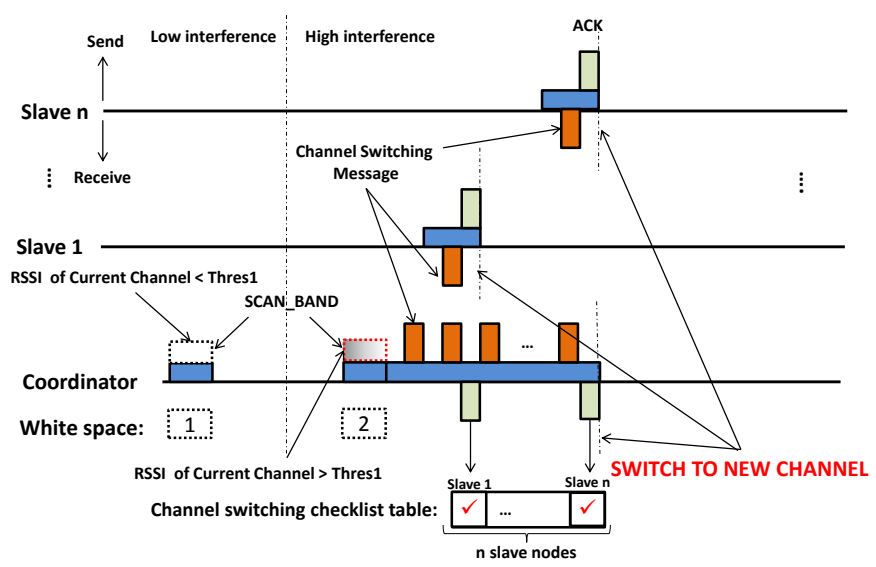

Fig. 5. Channel switching algorithm of C-RICER protocol.

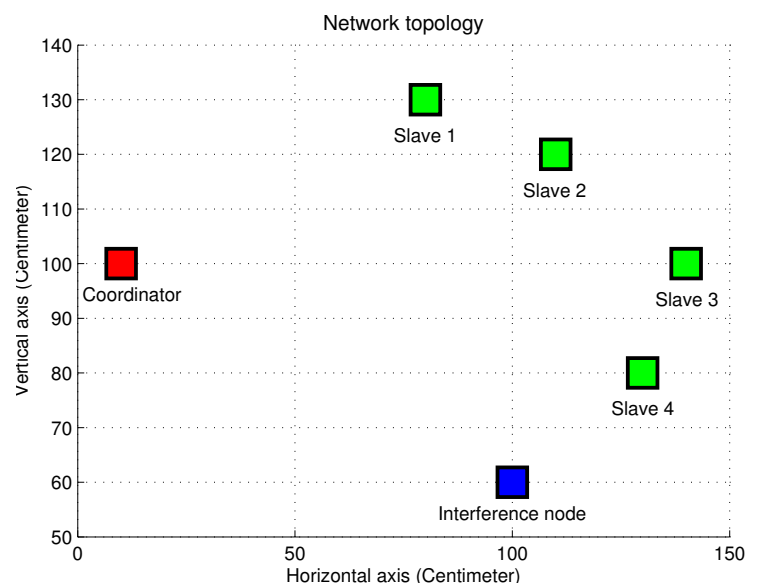

Fig. 6. Network topology.

waits for ACK message from slaves. With any successfully received ACK from a slave node, the Channel Switching Checklist Table of coordinator is filled at the position of that slave. When this checklist table is full, the coordinator will switch to the new channel.

If there is a packet to transmit, the slaves will wake up and wait for a wake up beacon message from the coordinator. Utilizing this characteristic, if a slave receives any channel switching message from coordinator during this time, it will send back the ACK message before switching to the new channel according to the channel information that is stored in channel switching message.

In some situation, the coordinator does not successfully receive the ACK message from a slave while that slave has already switched to the new channel. Therefore, the coordinator will also switch to the new channel even if its checklist table is not full after a period $T_{\text {wait checklist table. Moreover, }}$ a backup channel is also proposed for the slaves that lost the channel information in order to update the current working channel of the WBASN.

\section{Performance Evaluation}

The MiXiM wireless simulation framework on the foundation of OMNET++ simulator is used for the WBASN performance evaluation. At first, the original RICER3b protocol is re-simulated in MiXiM. Based on the RICER3b protocol, CRICER has been implemented including new functions such as multichannel simulation, channel sensing, spectrum agility and dynamically changing of transmission power. In this work, a network topology with one coordinator, four slaves and one interference node is set up to simulate a WBASN with the existence of interference as shown in Fig. 6. To limit the simulation time, only two channels are simulated. The simulation parameters are shown in TABLE I. To demonstrate the features of the cognitive task, two simulation scenarios are set up.

\section{A. Scenario 1: Spectrum agility performance}

In this scenario, the power adaptation feature is not used to concentrate on the great reaction of channel adaptation 


\begin{tabular}{|l|c|}
\hline Parameters & Values \\
\hline Simulation time & $500 \mathrm{~s}$ \\
\hline Transmit current for $P_{1}$ (default) & $17.4 \mathrm{~mA}$ \\
\hline Transmit current for $P_{2}$ & $19.7 \mathrm{~mA}$ \\
\hline Receive current & $19.7 \mathrm{~mA}$ \\
\hline Sleep current & $10^{-3} \mathrm{~mA}$ \\
\hline Channel sensing current & $19.7 \mathrm{~mA}$ \\
\hline Energy consumption per each channel switching [12] & $2 \mathrm{~mJ}$ \\
\hline Voltage & $3.3 \mathrm{~V}$ \\
\hline Transmission power $P_{1}$ (default) & $1 \mathrm{~mW}$ \\
\hline Transmission power $P_{2}$ & $2 \mathrm{~mW}$ \\
\hline RSSI threshold 1 (Thres1) & $10^{-4} \mathrm{~mW}$ \\
\hline RSSI threshold 2 (Thres2) & $2 \times 10^{-4} \mathrm{~mW}$ \\
\hline Sensing period & $0.5 \mathrm{~s}$ \\
\hline Normal scanning cycle ( $T_{\text {scan cycle }}$, default) & $10 \mathrm{~s}$ \\
\hline$T_{\text {beacon }}=T_{\text {buzz }}=T_{A C K}$ & $1.25 \mathrm{~ms}$ \\
\hline$T_{\text {data }}$ & $6.66 \mathrm{~ms}$ \\
\hline$T_{\text {switching message }}$ & $1.3 \mathrm{~ms}$ \\
\hline Time receiver wait for wake up beacon $\left(T_{\text {wait beacon }}\right)$ & $0.15375 \mathrm{~s}$ \\
\hline Time between two consecutive beacons & $0.15375 \mathrm{~s}$ \\
\hline Bit rate & $19200 \mathrm{bps}$ \\
\hline Length of data packet & $128 \mathrm{bits}$ \\
\hline Length of beacon, buzz and ACK message & $24 \mathrm{bits}$ \\
\hline Length of channel switching message & $28 \mathrm{bits}$ \\
\hline
\end{tabular}

TABLE I

SIMULATION PARAMETERS.

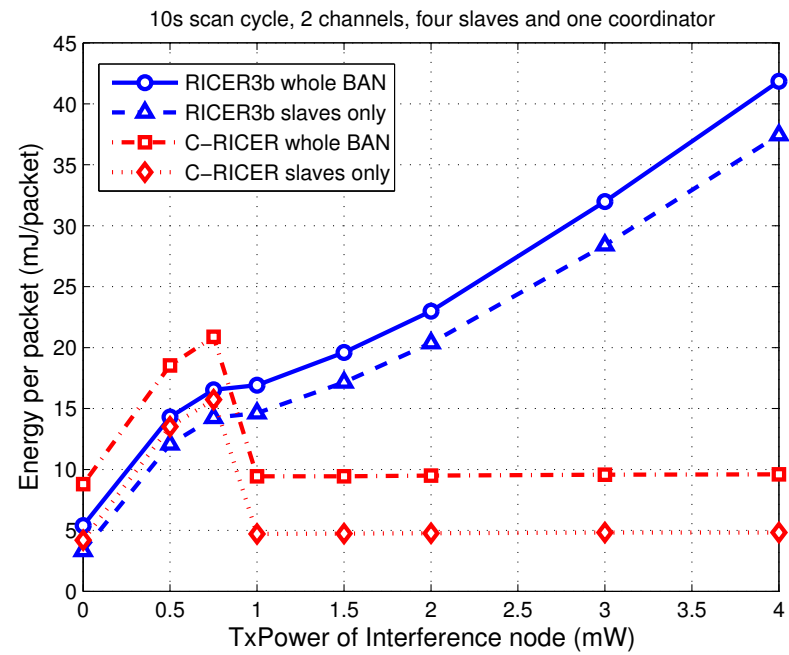

Fig. 7. Energy consumption per successfully received packets vs. interference power.

feature in high interference environment. The WBASN starts its operation in the interfered channel. An important point in the implementation of a CR network is the setting of the threshold. In a WBASN, the threshold is set according to the network topology and we set it equal to twice the received RSSI from the farthest slave. Therefore, the threshold in this case is selected as $10^{-4} \mathrm{~mW}$. This value is reached when the transmission power of the interference node is $1 \mathrm{~mW}$. This means that, the WBASN will immediately switch to a new channel when the transmission power of interference node is greater than or equal to $1 \mathrm{~mW}$.

Fig. 7 shows the energy per packet versus the transmission power of interference node. In this figure, the C-RICER pro-

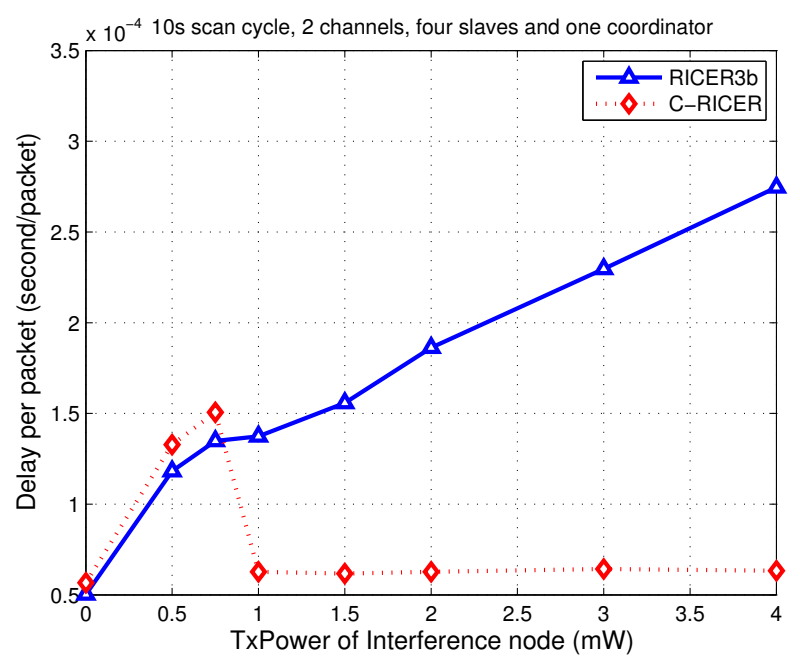

Fig. 8. Average delay per successfully received packet of WBASN.

tocol is simulated and compared with the traditional RICER3b protocol. The energy consumption is computed for both situations such that the coordinator is included (whole BAN) or not included (slaves only). As shown in the figure, when the RSSI of current working channel increases but its value is less than the threshold (i.e. TxPower equals $1 \mathrm{~mW}$ ), energy consumption of both protocols increases because the sensors frequently wake up for retransmission due to packet loss. The energy consumption of C-RICER at this time is more than RICER3b because of the periodic scanning for the interference level. However, when RSSI of current working channel reaches the threshold, the WBASN using C-RICER will switch to another non-interfered channel. Therefore, it can maintain the low energy per packet. In contrast, this metric of the WBASN using RICER $3 b$ rapidly rises up because it keeps working in the high interference channel.

Fig. 8 expresses the average delay per packet of WBASN. From the figure, the delay per packet of RICER3b quickly raises up during the increasing of interference. Conversely, CRICER can keep its packet delay metric steadily at a small value thanks to its cognitive mechanism.

\section{B. Scenario 2: Power adaptation performance}

This scenario simulates the adaptive changing of transmission power feature in a condition such that the interference only appears for a short period. To simulate that condition, the TxPower of the interference node is constantly set to 1.5 $\mathrm{mW}$ (the RSSI caused by this interference node is between Thres $_{1}$ and Thres $_{2}$ ). The interference node is set to work only 3 seconds after the first sensing of the coordinator. The initial working channel of whole WBASN and interference node is channel 1 .

Fig. 9 shows the comparison between C-RICER without power adaptation feature (1 TxPower) and C-RICER with power adaptation (2 TxPowers) for this scenario. The horizontal axis represents the increasing of the rescan cycle $T_{\text {rescancycle, }}$, while the vertical axis shows the energy con- 


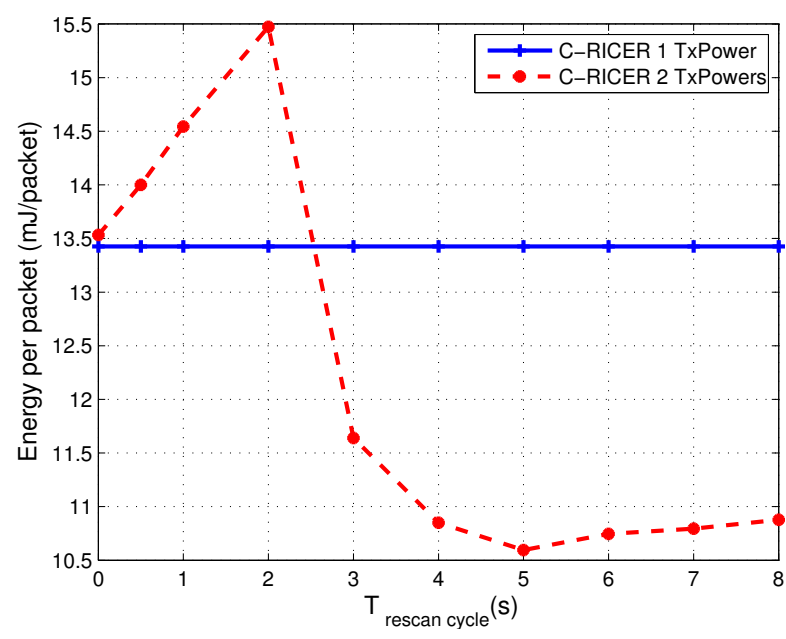

Fig. 9. The adaptive changing of TxPower feature evaluation.

sumption per successfully received packets of all slaves in the network. In C-RICER with 1 TxPower, after finishing the channel sensing, it will switch to the new channel. However, C-RICER with 2 TxPowers will change its TxPower to $P_{2}$ and rescan the band in the next $T_{\text {rescan cycle }}$ seconds. Therefore, when the $T_{\text {rescan cycle }}$ is less than the duration of interference (3 seconds), it means that the interference node is still working at the time the coordinator rescans the channel, then the WBASN will decide to switch its working channel. The energy consumption will be the total of the energy for channel switching and the energy for changing TxPower. For that reason, the energy per successfully received packets of CRICER with 2 TxPowers is higher than that of C-RICER with 1 TxPower in this region.

However, when the $T_{\text {rescan cycle }}$ is greater than the duration of interference, the interference disappears at the time the coordinator rescans the band. Therefore, the WBASN will change its TxPower back to $P_{1}$ and continue to work in the current channel instead of switching the channel. Moreover, using the high transmission power during $T_{\text {rescan cycle }}$ increases the number of successfully received packets. For that reason, the energy per successfully received packets of C-RICER with 2 TxPowers is less than that of C-RICER with 1 TxPower in this region. It is also shown in this figure that the energy per packet of C-RICER with 2 TxPowers also raises up with the increasing of $T_{\text {rescan cycle }}$.

In summary, the value of $T_{\text {rescancycle }}$ should be long enough to cover a short duration or interference, but short enough to ensure the high transmission power is not used for too long to save the energy. As an extension for the future work, the duration of short interference could be predicted using machine learning method. Hence, $T_{\text {rescan cycle }}$ could be adaptively selected based on this value.

\section{CONCLUSION}

Wireless technology plays an important role in the modern life. However, the densely distribution of personal wireless devices leads to a problem of crowded wireless spectrum in the open frequency bands. The performance of WBASNs then decreases in those bands because of the coexisting interferences. This paper proposes C-RICER, a protocol with cognitive functions to avoid the interference and enhance the performance of WBASNs. The simulation results show that the C-RICER protocol is able to outperform RICER3b under high interference environment, especially in terms of energy consumption.

Recently, in February 2013, IEEE Standards Association has putted an adjustment to the IEEE 802.15.4j standard in terms of physical layer extension to support WBASN services operating in the $2360-2400 \mathrm{MHz}$ band for medical applications [13]. The proposed C-RICER MAC protocol could be also used by WBASNs to dynamically work in this frequency band. C-RICER can detect and avoid the coexistence with primary users of this band thanks to its cognitive function.

\section{REFERENCES}

[1] M. A. Hanson, H. C. Powell Jr., A. T. Barth, K. Ringgenberg, B. H. Calhoun, J. H. Aylor, and J. Lach, "Body area sensor networks: Challenges and opportunities," IEEE Computer, vol. 42, no. 1, pp. 58-69, 2009.

[2] L. Sydanheimo, M. Keskilammi, and M. Kivikoski, "Performance issues on the wireless $2.4 \mathrm{GHz}$ ISM band in a multisystem environment," IEEE Transactions on Consumer Electronics, vol. 48, no. 3, pp. 638-643, 2002.

[3] J. Mitola III and G. Q. Maguire Jr, "Cognitive radio: making software radios more personal," IEEE Personal Communications, vol. 6, no. 4, pp. 13-18, 1999.

[4] S. Haykin, "Cognitive radio: Brain-empowered wireless communications," IEEE Journal on Selected Areas in Communications, vol. 23, no. 2, pp. 201-220, 2005.

[5] S. A. Gopalan, D.-H. Kim, J.-W. Nah, and J.-T. Park, "A survey on power-efficient MAC protocols for wireless body area networks," in 3rd IEEE International Conference on Broadband Network and Multimedia Technology, 2010, pp. 1230-1234.

[6] K. A. Ali, J. H. Sarker, and H. T. Mouftah, "A mac protocol for cognitive wireless body area sensor networking," in Proceedings of the 6th International Wireless Communications and Mobile Computing Conference. ACM, 2010, pp. 168-172.

[7] A. De Domenico, E. C. Strinati, and M.-G. Di Benedetto, "A survey on MAC strategies for cognitive radio networks," IEEE Communications Surveys and Tutorials, vol. 14, no. 1, pp. 21-44, 2012.

[8] B. Latré, B. Braem, I. Moerman, C. Blondia, and P. Demeester, "A survey on wireless body area networks," Kluwer Academic Publishers Wireless Networks, vol. 17, no. 1, pp. 1-18, 2011.

[9] A. Bachir, M. Dohler, T. Watteyne, and K. K. Leung, "MAC essentials for wireless sensor networks," IEEE Communications Surveys \& Tutorials, vol. 12, no. 2, 2010.

[10] E.-Y. Lin, J. M. Rabaey, and A. Wolisz, "Power-efficient rendez-vous schemes for dense wireless sensor networks," in IEEE International Conference on Communications, vol. 7, 2004, pp. 3769-3776.

[11] E.-Y. Lin, J. M. Rabaey, S. Wiethoelter, and A. Wolisz, "Receiver initiated rendezvous schemes for sensor networks," in IEEE Global Telecommunications Conference, vol. 5, 2005, pp. 3117-3122.

[12] C. Haiming, L. Cui, and S. Lu, "An experimental study of the multiple channels and channel switching in wireless sensor networks," Technical Report WSN-ICT-CAS-20090502, 2009.

[13] IEEE Standards Association, "IEEE Standard for Local and metropolitan area networks Part 15.4: Low-Rate Wireless Personal Area Networks (LR-WPANs), Amendment 4: Alternative Physical Layer Extension to Support Medical Body Area Network (MBAN) Services Operating in the 2360 to $2400 \mathrm{MHz}$ Band," Technical Report IEEE Std 802.15.4j, February, 2013. 